\title{
SOLITAIRE: A new generation solenoidal fusion product separator
}

\author{
M.D. Rodríguez, M.L. Brown, M. Dasgupta *, D.J. Hinde, D.C. Weisser, T. Kibèdi, M.A. Lane ${ }^{1}$, P.J. Cherry ${ }^{2}$, \\ A.G. Muirhead, R.B. Turkentine, N. Lobanov, A.K. Cooper, A.B. Harding, M. Blacksell, P.M. Davidson ${ }^{3}$
}

Department of Nuclear Physics, Research School of Physics and Engineering, The Australian National University, ACT 0200, Australia

\section{A R T I C L E I N F O}

Article history:

Received 4 September 2009

Received in revised form

24 November 2009

Accepted 10 December 2009

Available online 22 December 2009

Keywords:

Superconducting solenoid

Gas filled separator

Evaporation residues

Fusion cross-sections

\begin{abstract}
A B S T R A C T
A superconducting solenoidal fusion product separator, based on a $6.5 \mathrm{~T}$ solenoid, has been developed at the Australian National University to enable separation and detection of evaporation residues following heavy-ion fusion reactions. This device, with an angular coverage of $0.45-9.5^{\circ}$, produces a spatial separation between the fusion products and the intense background of elastically scattered beam particles. Its high efficiency allows precise measurement of nuclear fusion cross-sections, as well as being ideal for evaporation residue coincidence measurements. The essential features of the system and the first results obtained are described.
\end{abstract}

(c) 2009 Elsevier B.V. All rights reserved.

\section{Introduction}

Amongst the most significant advances in understanding fusion of heavy nuclei is the realization that two colliding nuclei encounter not just a single fusion barrier, but can be thought of as experiencing a distribution of fusion barrier energies, determined by the structure of the two colliding nuclei [1,2]. High precision fusion cross-sections have been used to determine the experimental distribution of barriers [3-5], which have clearly demonstrated the role of nuclear deformation and complex surface vibrations in nuclear fusion. This realization has also provided a new avenue to understand the relationship between entrance channel properties and the subsequent evolution of the dinuclear system towards an equilibrated compound nucleus [6]. This is a subject of intense current interest, as efforts continue to map out a route towards the centre of the island of super-heavy elements.

Underpinning these results and advances has been the ability to make precise and accurate measurements of cross-sections and characteristics of evaporation residues (ERs) and fission fragments, which are the product nuclei formed following a nuclear fusion reaction. The measurement of fission fragments is rather simpler than measurement of ERs, as two fragments are present, distributed over all angles, and coincidence techniques can be

\footnotetext{
* Corresponding author.

E-mail address: Mahananda.Dasgupta@anu.edu.au (M. Dasgupta).

${ }^{1}$ Current address: 27 Luehmann St, Page, ACT 2615, Australia.

${ }^{2}$ Current address: Bytemark Hosting, The Raylor Centre, James Street, York, UK.

${ }^{3}$ Current address: Research School of Earth Sciences, The Australian National University, ACT 0200, Australia.
}

used to remove background events and obtain reliable crosssections. Evaporation residues can be measured either by direct detection of the ERs themselves, or (if unstable) by indirect methods in which their subsequent decay (for example $\gamma$-decay or $\alpha$-decay) is detected. The direct detection method can be used for almost all fusion reactions, and has been widely applied. However, since evaporation residue yields are peaked around $0^{\circ}$, they have to be physically separated from both the direct beam, and the intense flux of elastically scattered beam particles. Separation has been achieved using electric and/or magnetic fields, and a variety of devices employing such techniques have been developed in the past $[7,8]$. However, it is difficult to achieve both high accuracy and precision, which requires large (or well known) solid angle coupled with high transmission efficiency. The first condition is particularly difficult to achieve for electromagnetic separators which typically have $\approx 4-10 \mathrm{msr}$ solid angle and complex beam optics $[7,8]$. A separator based on a superconducting solenoid, with a solid angle of $86 \mathrm{msr}$, has been developed at the Australian National University for making precision measurement of ERs. This paper describes the 6.5 T superconducting gasfilled solenoid for in-beam transport and identification of recoiling evaporation-residues, called SoliTAIRE, and presents the first fusion excitation function measurements made using this device.

\subsection{The ideal separator}

The requirements of an ideal separator for modern fusion measurements are demanding, as it is desirable to make measurements having a precision better than $1 \%$, and accuracy 
approaching that value. The former requires high statistics, whilst the latter requires a very well-known ER transmission efficiency. Evaporation residues, though peaked in the beam direction, are spread in angle by the recoil resulting from the evaporation of light particles (and by multiple-scattering in the target). There are two approaches to handle this problem. The angle of the separator can be scanned to span the angular range of the ERs $[9,10]$ and thus determine the angular distribution. As an example, the compact velocity filter developed at the Australian National University [9] for measurements of fusion barrier distributions has a transmission efficiency of $98-100 \%$. However, the small angular acceptance necessary for clean separation of ERs and beam particles meant that time-consuming angular distributions had to be measured, and high statistics could only be achieved by relatively long counting times. The alternative approach is to develop a separator accepting essentially the whole angular range of the ERs, allowing the rapid collection of high statistics. However, this implies that the ER angular distribution must already be known in order to obtain the correct transmission efficiency of the device, which is required for high accuracy. Although angular distributions can be estimated with a statistical model evaporation code, this could lead to unacceptable uncertainties in the transmission efficiency. An ideal separator should have a large angular acceptance (ideally accepting as close to $100 \%$ of all ERs as possible), a high transmission efficiency, and simultaneously provide information on the experimental angular distribution to allow reliable calculation of the transmission efficiency. Such a separator would also be ideal for coincidence measurements, both for detectors viewing the primary target, and at the secondary focus. Not only would it give a high coincidence yield, but an acceptance close to $100 \%$ would minimize bias in the coincidence data due to different efficiencies for different products. As described below, a separator based on a solenoid can satisfy all these requirements.

The principle of operation of a solenoidal separator is discussed in the next section, which is followed by a description of the features specific to Solitaire. The experiments carried out to optimize the performance of Solitaire, such as the choice of filling gas, study of charge states in gas, and the determination of image size are described in Section 3. The method of detecting and identifying evaporation residues, and the first cross-section measurements using Solitaire are discussed in Sections 4 and 5, respectively, followed by a discussion summarizing the boundaries of the use of the device in Section 6.

\subsection{Solenoid optics}

Solenoids have good focusing properties with small geometrical aberrations [11,12]. Provided beam particles can be efficiently rejected, these properties, combined with a large solid angle, make solenoids an ideal separating element for ERs. The optical properties of solenoids are well understood [11-13], and are summarized below.

The optical properties of an infinite solenoid are extremely simple. Due to the azimuthal symmetry of solenoids, cylindrical coordinates are appropriate to describe the optics of the device. A particle of mass $m$ emitted from the axis at an angle $\theta$ will interact with the magnetic field (which for a solenoid of infinite length only has an axial component $B_{z}$ ), through the radial component $v_{r}$ of its velocity $v$, experiencing a normal force $q v_{r} B_{z}$. It will follow a circular trajectory in the plane perpendicular to the axis, and will thus return to the axis in the time taken to complete exactly one turn, subsequently continuing on its helical trajectory. The distance traveled along the axis $(z)$ thus depends only on the component of velocity along the axis $v_{z}$ through the expression $z=2 \pi m v_{z} / q B_{z}$, and thus the focal length $f$ is given by $f=\pi m v_{z} / 2 q B_{z}$. Since the $z$-component of velocity is $v_{z}=v \cos \theta$, if the particles of interest are emitted only at small $\theta$, then $v_{z} \approx v$, and the focal length is to first order independent of $\theta$.

SolitAIRE is of course a finite solenoid, with the particles emitted from the axis and returning to the axis outside the region of strong magnetic field. In this case, the trajectories are not quite so simple. Taking only the velocity and field components considered in the case of the infinite solenoid, the particle would not return to the axis after passing through the solenoid. However, on entering and leaving the solenoid, the particle experiences the radial component of the magnetic field $\left(B_{r}\right)$, which interacts with the axial component of the velocity, to generate an additional force on the particle. Busch's theorem shows that the particles come back to the axis, almost independent of $\theta$, as in the case of an infinite solenoid. In 1926 and $1927 \mathrm{H}$. Busch [14,15] demonstrated the similarities between optical and magnetic lenses. The solenoid acts as a thin converging lens with a focal length $f$ given in the first order paraxial approximation by [16]

$f \approx 4 \frac{p^{2}}{q^{2}} \frac{1}{\overline{B_{z}^{2}} L}$

where $p$ is the momentum of the particle, $L$ is the length of the solenoid, and $\overline{B_{z}^{2}}$ is the average value of the square of the axial magnetic field $B_{z}$. An important point to note is that a solenoid is not mass-dispersive, but dispersive in momentum and charge. It is vital to recognise that forward-scattered beam particles and the ERs formed following complete fusion leave a thin target with the same average momentum, thus they are only separated if their charge states are different.

\subsection{Gas-filled solenoid}

Elastically scattered particles and ERs emerge from the target each with their characteristic distribution of charge states. If these distributions overlap, the trajectories of elastics and ERs through the solenoid will overlap, and full separation will not be achieved. By filling the magnetic field region with gas, the ions will undergo collisions in which their charge state is changed by loss or capture of electrons [17-20]. With a sufficient number of charge-changing collisions, their trajectories can be characterized as those of particles having their mean or equilibrium charge states [21]. The latter depends on the atomic number $Z$ and velocity of the ion [21-23]. In general, the smaller velocity of ERs results in their average charge state being lower than that of the beam particles. If this charge difference is sufficient, they will have different average trajectories, and according to Eq. (1), will come to a focus at different points on the axis. This can allow the elastically scattered beam particles to be physically blocked. Particles travelling parallel to the solenoid axis, however, cannot be separated from the elastically scattered beam. Although immediately after fusion the ERs travel at $0^{\circ}$, the recoil effects from the evaporation of particles after fusion generally deflect the ERs away from $0^{\circ}$ (as can multiple-scattering in the target). Thus by blocking the direct beam before it enters the solenoid, only a relatively small fraction of ERs is lost. Since a solenoid accepts all azimuthal angles, the maximal acceptance of the $\mathrm{d} \sigma / \mathrm{d} \theta$ distribution is desired. Since the $\mathrm{d} \sigma / \mathrm{d} \theta$ distribution is zero at $0^{\circ}$, a cup subtending an angle of $<1^{\circ}$ stops typically a few $\%$ of the ERs. The situation is completely different for devices with a small solid angle where the quantity $\mathrm{d} \sigma / \mathrm{d} \Omega$, which peaks at $0^{\circ}$, needs to be maximal for best ER acceptance. Thus most separators, which have smaller solid angles, accept the direct beam and the ERs around $0^{\circ}$. 


\section{Basic features of SOLITAIRE}

The initial design of Solitaire was guided by calculations using estimates of equilibrium charge states of ERs and beam particles in gas. From these, a maximum field of $6.5 \mathrm{~T}$, a coil length of $60 \mathrm{~cm}$, and a warm-bore diameter of $20 \mathrm{~cm}$ gave a good compromise between performance and size (and thus cost). In practice, the charge states proved somewhat different from estimations, giving both advantages and disadvantages, as discussed in Section 3. The superconducting solenoid was manufactured by Cryogenics Ltd., UK. It is cooled by a Sumitomo cryocooler with a base temperature of $3.2 \mathrm{~K}$, having $1 \mathrm{~W}$ of cooling power at that temperature. The coil is mounted in a vacuum enclosure and surrounded by a super-insulated radiation shield crycooled to $50 \mathrm{~K}$. The device takes $60 \mathrm{~h}$ to cool from room-temperature to operational temperature. The axial and radial position of the cryostat inside the iron yoke (see Section 2.2) can be adjusted by external means. The axial movement allows the elimination of out-of-balance forces on the solenoid, which are measured using load cells between the solenoid and the iron yoke. The adjustment of the radial position enables the alignment of the magnetic axis of the solenoid with the beam axis.

The separator system SoltaiRE consists of the target chamber, the solenoid surrounded by the soft-iron yoke, and the detector chamber, as indicated in the external view of the device shown in Fig. 1. The vacuum chambers and other movable components either side of the solenoid are mounted on trolleys running on axial rails, to simplify access to internal components during experimental setup.

A cross-sectional view of Soltaire is given in Fig. 2. The target chamber and solenoid bore can be filled with low-pressure gas. The accelerator beam line is isolated from the gas-filled target chamber by a thin carbon foil $9 \mathrm{~mm}$ in diameter, through which the beam passes. The beam, $\approx 1.5 \mathrm{~mm}$ in diameter, after entering the target chamber passes through the target and then is stopped in a $5 \mathrm{~mm}$ diameter Faraday cup (FC).

Three main classes of particles enter the magnetic field region beyond the target. These are the primary beam, which is stopped in the FC, elastically scattered beam particles, and the ERs. The scattered beam particles and ERs emitted at angles larger than $0.45^{\circ}$ subtended by the FC continue on their trajectories into the magnetic field region. A set of axial blocking discs is placed at the focus of the scattered beam particles, which prevents them from reaching the detector chamber. The ERs, due to their lower average charge state, reach a focus beyond these discs, entering the detector chamber. There they can be identified by their timeof-flight with respect to the beam pulse and their energy or energy-loss signal in a detector. The flight path from the target to the detector can be varied between 1.35 and $1.95 \mathrm{~m}$, depending on requirements. This is a very short path compared to most separators, and has been exploited in recent applications of SoltTAIRE to nuclear structure studies of short-lived isomers [24].

A unique feature of Soltaire is that the solenoid is enclosed in a $2400 \mathrm{~kg}$ soft-iron return yoke to minimize the external field, which is reduced to only a few $\mathrm{mT}$ (see Fig. 2). The yoke was designed inhouse using the code POISSON/SUPERFISH [25], such that at the maximum magnetic field, the field in the iron is approaching saturation. As a result the ion trajectories in the target chamber preceding the solenoid, and in the detector chamber following the solenoid, are essentially unaffected by the magnetic field of the solenoid. A secondary advantage of the iron yoke is that there are no restrictions on working near the solenoid even when it is at maximum field. The details of each component of Soltaire are described below.

\subsection{Target chamber and gas cell}

The target chamber houses the entry window and the target ladder, as well as having space reserved for future placement of

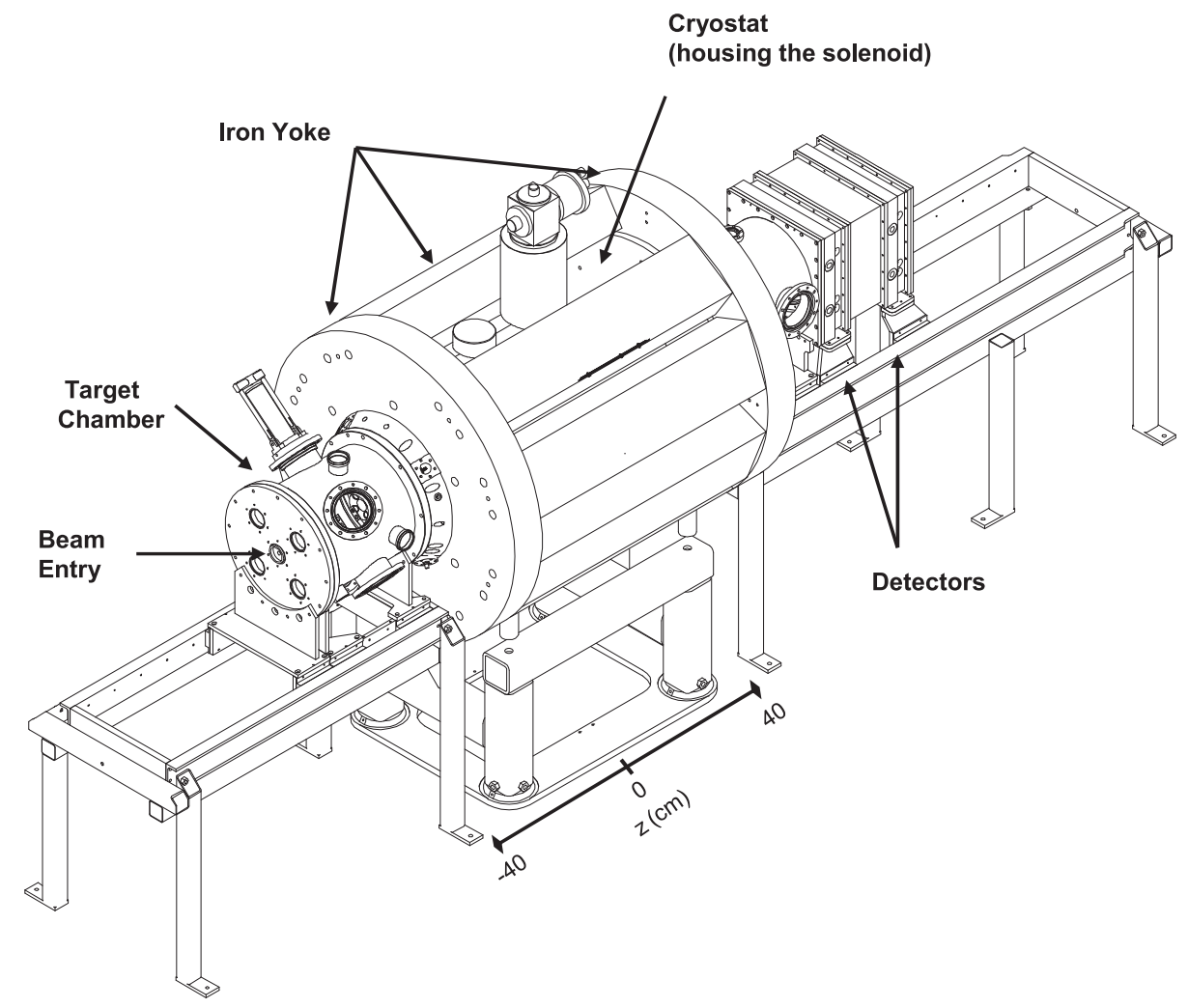

Fig. 1. External view of Solitaire. 

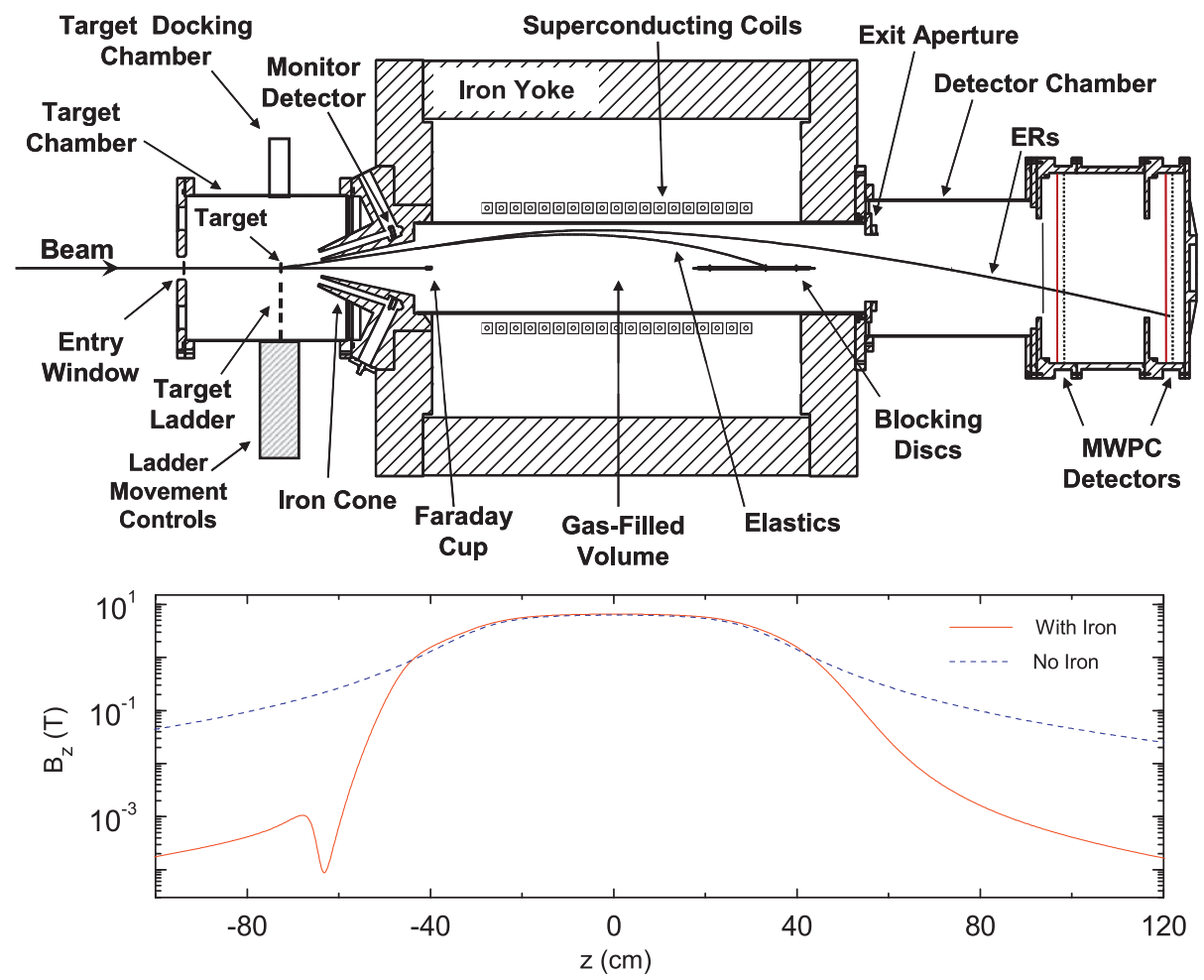

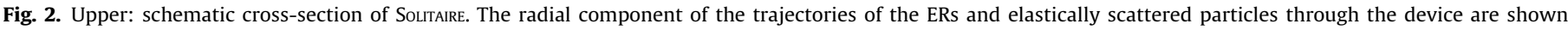

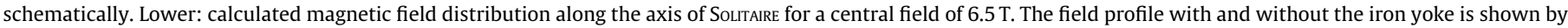
the full and dashed lines, respectively.

detectors to view the target at both backward and forward angles. The beam entry window is located at the up-stream end of the target chamber. It isolates the high vacuum $\left(\sim 10^{-7}\right.$ Torr $)$ of the accelerator beam line from the target chamber and the solenoid bore, which are filled with the operating gas (typically 1 Torr He, see Section 3.1). Up to six windows, mounted on a rotary cassette, can be exchanged using an external actuator allowing easy testing for vacuum-tightness. Since the direct beam passes through the window, a robust material must be used. We have successfully used evaporation-deposited carbon foils between 14 and $65 \mu \mathrm{g} / \mathrm{cm}^{2}$ in thickness. One foil lasts for many days, indeed we have not experienced a beam-related window failure.

The linear target ladder, holding up to six targets, is moved by a computer-controlled external stepper motor. To replace targets, the ladder is driven into a cylindrical target dock chamber. The ladder shaft itself seals the chamber, which can then be let up to atmosphere and evacuated independently of the rest of the device.

The target chamber is connected to the nickel-plated soft-iron cone, which in turn is connected to a $20.5 \mathrm{~cm}$ diameter, $80 \mathrm{~cm}$ long thin-walled aluminium tube. This connects with the detector chamber behind the solenoid, thus forming a single vacuum space or gas cell, which during operation is filled with low pressure gas. A gas flow system keeps the gas at a constant pressure and prevents the build up impurities from outgassing. It is also possible to isolate the detector chamber from the gas cell using a thin window if required.

\subsection{Return magnetic circuit}

The soft-iron yoke (made from 1010 low-carbon steel) provides a low impedance return path for the magnetic field. In addition, a hollow soft-iron cone weighing $115 \mathrm{~kg}$ is placed at the entrance to the solenoid. This cone, extending towards the target, reduces the magnetic field in the target region to $\sim 1 \mathrm{mT}$ as shown in the lower part of Fig. 2. It also minimizes the field along the path taken by elastically scattered beam particles travelling to the monitor detectors. The four monitor detectors are symmetrically located in separate cylindrical cavities machined into the iron cone. These each intersect another cylindrical cavity pointing at the target at $18^{\circ}$ with respect to the beam axis. As a result of the surrounding soft iron, the maximum magnetic field in the cylindrical apertures is around $1.5 \mathrm{mT}$, when the field in the iron is about $1 \mathrm{~T}$. Such a cone cannot be used at the other end of the solenoid, since in some reactions, the desired fusion products may exit the solenoid with their focus at infinity (i.e. with their trajectories parallel to the axis of the solenoid).

The maximum angular acceptance of Solitaire, $\theta=10.3^{\circ}$, is determined by the entrance aperture of the cone. In practice, trajectories of ERs strike the inner wall of the vacuum tube which passes through the warm bore if emitted at angles greater than $\sim 9.5^{\circ}$. Thus the practical upper limit of the angular acceptance is $9.5^{\circ}$.

\subsection{The Faraday cup}

The function of the Faraday cup is to stop the direct beam and the most intense forward-angle scattered beam particles, thus suppressing the largest source of undesired particles from reaching the detector. A $5 \mathrm{~mm}$ diameter Faraday cup made from tantalum suspended by two crossed $0.1 \mathrm{~mm}$ diameter stainless steel wires is located on-axis inside the cone. It subtends $0.45^{\circ}$, however, discs of larger diameter can be attached concentrically, to increase the angle subtended. Since the beam from the tandem accelerator at the ANU has a typical FWHM of $1 \mathrm{~mm}$, the Faraday cup alone is sufficient to stop the direct beam. Beams with large spatial spread would require a bigger Faraday cup, with a consequent drop in ER transmission. 


\subsection{Blocking discs and rod}

The beam particles at angles greater than that subtended by the Faraday cup travel through the solenoid. Typically, due to their higher average charge state in gas, they are focused back onto the axis closer to the target than are the ERs. An axial threaded rod, supported by $0.1 \mathrm{~mm}$ stainless steel wires, and a set of moveable discs block these particles (Fig. 2). The 6 or $12 \mathrm{~mm}$ diameter discs can be located at any point along the $25 \mathrm{~cm}$ rod, or removed completely, depending on the needs of the experiment. The optimal location of the discs to suppress beam particles, yet intercept as few ERs as possible, is estimated before the experiment through calculation of the trajectories of both the ERs and the beam particles. The calculations are done using a Monte Carlo ray tracing code, SOLIRTE [26], developed in house to simulate the ion's trajectories through the magnetic field, including scattering and charge exchange interactions with the filling gas.

\subsection{Exit aperture}

An aperture can be placed at the exit of the solenoid bore, to restrict the particles which are allowed to enter the detector chamber. The aperture can be chosen, according to the experiment, up to $20 \mathrm{~cm}$ in diameter. A gas window $(0.7 \mu \mathrm{m}$ PET foil $)$ isolating the detector chamber from the gas volume can be mounted across this aperture.

\subsection{Detector chamber}

The axis of the cylindrical detector chamber, with a diameter of $32 \mathrm{~cm}$, coincides with that of the solenoid. It is mounted on rails that permit easy opening and access. It is pumped by a turbopump that moves with the chamber. The chamber has been used to mount $\mathrm{Si}$ detectors for tests of implantation-decay measurements [27]. The main detector system of SoltTAiRE is a pair of multiwire proportional counters (MWPC) which are mounted from the back flange of the detector chamber.

\section{7. $M W P C$ detectors}

The MwPC detectors give position sensitivity, good timing, high count rate capability and have large area. The high count rate capability results from the short electron and ion drift times arising from the high electric field and a small distance between cathode (foil) and anodes (grids). The $20 \mathrm{~cm} \times 20 \mathrm{~cm}$ MWPC detectors built for SOLITAIRE consist of two position sensitive wire grids, (one aligned horizontally and one vertically), and a central foil, operating in 3.5-4 Torr of propane gas. Each grid consists of 200 parallel $20 \mu \mathrm{m}$ gold coated tungsten wires, spaced by $1 \mathrm{~mm}$. These grids are placed $3 \mathrm{~mm}$ either side of the central cathode, a $0.7 \mu \mathrm{m}$ polyethylene terephthalate (PET) foil, with $40 \mu \mathrm{g} / \mathrm{cm}^{2}$ of gold deposited on both sides. The cathode is divided into three electrically isolated sections to reduce capacitance. The signals from the foil (with a rise-time of $\sim 2 \mathrm{~ns}$ ) are the trigger for the тоғ measurement as well as providing the energy loss $(\Delta E)$ signal from the MWPC. The position resolution of the MWPC is $\sim 1 \mathrm{~mm}$, which is smaller than the size of the primary beam spot. The geometrical efficiency of a single MWPC is $98 \%$, due to the position wires of the first grid blocking $2 \%$ of the detection area. Signals from ERs entering the detector are well above the electronic thresholds. A $0.7 \mu \mathrm{m}$ PET window, $20 \mathrm{~cm}$ in diameter, separates the detector gas volume from the detector chamber. A gas flow system maintains a constant pressure, and renews the gas, preventing buildup of contamination resulting from out-gassing of the detectors.

The full detector configuration consists of two MwPC detectors in a single gas cell, normally located $24 \mathrm{~cm}$ apart, with the first detector placed a distance of $168 \mathrm{~cm}$ from the target. The two detectors allow velocity determination without requiring a pulsed beam, and also the determination of the trajectories of particles passing through both detectors. The image on the second detector is broadened due to the scattering in the gas-window, the central foil of the first detector, and the gas in the space between the two detectors. However, for the reactions described here, the image size of the ERs is much smaller than the active area of the second detector, and thus the small spreading of the image due to scattering does not affect the measurements in the second MWPC. The second MwPC detects 96\% of all the ERs measured in the first detector, which is consistent with the expected loss of ERs that hit the wires of the position grid. The energy loss suffered by the ERs before reaching the second detector, proved to be beneficial in separating the ERs from the beam degraded particles, as described in Section 4.

\subsection{Si detectors}

A silicon detector has been tested for identifying ERs in the SOLITAIRE detector chamber. As the detector area was smaller than the MWPC, it was placed upstream of the MWPC, at the focus of the ERs. It provides total energy and timing signals, however, for ER identification, it was found that use of the Si detector did not give a significantly cleaner spectrum than the use of two mwPCs. The detector was also used to successfully test the implantation-decay method of ER identification, measuring $\alpha$-decays between beam bursts [27].

\section{Performance of SOLITAIRE}

Several experiments were carried out to optimize the conditions to measure fusion excitation functions with SolitalRe. The key performance criteria for such a device are the transmission efficiency of the desired nuclei (ERs), and the rejection of unwanted events (generally beam particles). Different variables were investigated to optimize the operating conditions, and the physical configuration of blocking discs and apertures. Simulations predicted up to $90 \%$ of all ERs leaving the target could be transmitted to the detectors, for favourable reactions.

\subsection{Operating gas}

The equilibrium charge state $\langle q\rangle$ of the ions travelling through a gas depends on the identity of the gas and the velocity and atomic number of the ions. Argon, helium, neon, nitrogen and propane gases were tested, with pressures up to 3 Torr. The best ER transmission efficiency and ratio of ERs to beam particles, for the two test reactions $\left({ }^{32} \mathrm{~S}+{ }^{89} \mathrm{Y}\right.$ and $\left.{ }^{58} \mathrm{Ni}+{ }^{58} \mathrm{Ni}\right)$, was obtained with helium at approximately 1 Torr. The rejection of beam particles for the latter reaction was unexpectedly better than had been anticipated. It seemed likely that this was related to the charge state of the beam particles in the gas, thus measurements were carried out to investigate this behaviour.

\subsection{Beam particle charge states}

The trajectories and the charge distributions of ${ }^{58} \mathrm{Ni}$ ions scattered from a ${ }^{58} \mathrm{Ni}$ target were determined at energies near the fusion barrier $\left(\mathrm{V}_{B}\right)$, to investigate the change in the charge state 
distribution of ${ }^{58} \mathrm{Ni}$ ions as a function of the He gas pressure along their path.

A collimator with a pattern of nine small circular apertures was placed at the entrance of the iron cone. The image of this pattern on the MwPc detector was obtained as a function of the gas pressure (varied from 0 to 3 Torr), and the magnetic field, scanned from 3 to $5.5 \mathrm{~T}$. With no gas, the charge state distribution through the solenoid is essentially unchanged from that on exiting the target. Thus following Eq. (1), one aperture in the collimator gives rise to several images on the detector, one for each charge state.

The measured $\mathrm{x}-\mathrm{y}$ position spectra for elastically scattered beam particles, obtained at $E_{\text {Lab }}=220 \mathrm{MeV}\left(1.1 \mathrm{~V}_{B}\right)$, are shown in Fig. 3(a) and (c). The circular images on a curved radial locus correspond to the passage of different charge states through one of the apertures. To verify the trajectory calculations of the simulation code soLIRTE (without yet considering charge exchange in the gas), these position spectra were compared with the code predictions. As seen by comparing Fig. 3(b) and (d) with the experiment, the agreement is very good. As the magnetic field increases, the elastically scattered particles are imaged closer to the exit of the solenoid. Therefore the position of each charge state moves to a larger radius on the detector, as shown in Fig. 3(c).

With the introduction of gas, the elastically scattered beam particles undergo charge-changing collisions and/or scattering with the gas molecules. At typical gas pressures of few Torr used for the ER measurements, only a few of the elastically scattered beam particles suffer charge-changing collisions. Thus, the charge state distribution and trajectories of the elastics remain essentially unchanged after going through the gas, except that the trajectories are diffused due to scattering collisions, and the few charge-changing interactions with the gas. Thus, the image on the detectors, shown in Fig. 4(a) and (c) for gas pressures of 1.5 and 3.0 Torr, respectively, is essentially a more diffuse version of Fig. 3(c). The predictions made by the code are shown on the right panels, and are in good agreement with the experimental data. The comma-like loops between the main charge state clusters (most apparent in Fig. 4(b), indicated by the arrow) are caused by particles which undergo a single charge exchange event at some point in their progress through the solenoid. One side of the loop corresponds to electron loss events from lower to higher charge state, and the other side corresponds to electron capture from higher to lower charge state. For the innermost groupings, their low charge state means that the electron loss (arrowed) is strongly preferred, and the electron capture is barely visible.

\subsection{Average charge state of ERs}

One of the first measurements carried out was to determine the average charge state of ERs in He gas. The ER image size on a single MwPC detector was determined as a function of magnetic field. A beam of $240 \mathrm{MeV}{ }^{58} \mathrm{Ni}$ was incident on a range of thin elemental or isotopically enriched targets from $Z=28$ to 46 . The ERs were identified as described in the next section. The ER position distribution in the plane perpendicular to the beam axis was projected onto one axis, and fitted with a Gaussian function. The measured FwHM as a function of magnetic field is shown in Fig. 5. The minimum image size corresponds to the focus of the ERs being located at the axial position of the detector. The mean ER charge state can then be determined by matching the focus of the ERs (minimum image size) with simulations. The accuracy of phenomenological models can then be estimated by comparing
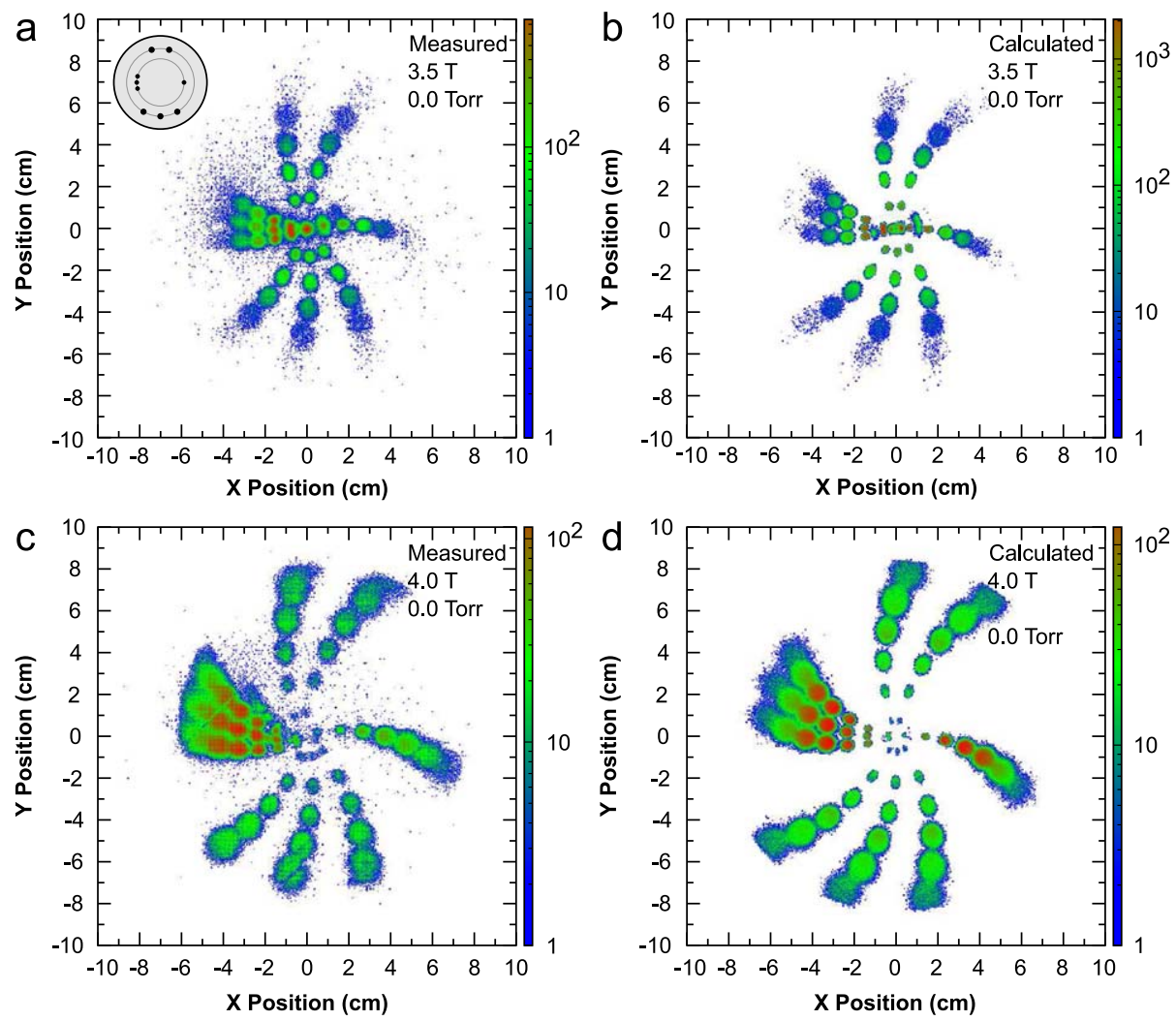

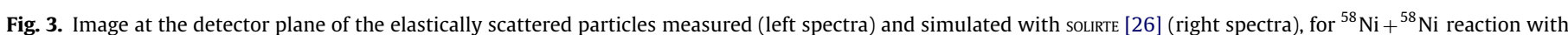
$E_{L a b}=220 \mathrm{MeV}$, at different $B_{z}$ with no gas in the cell. The collimator pattern is shown in panel (a). 

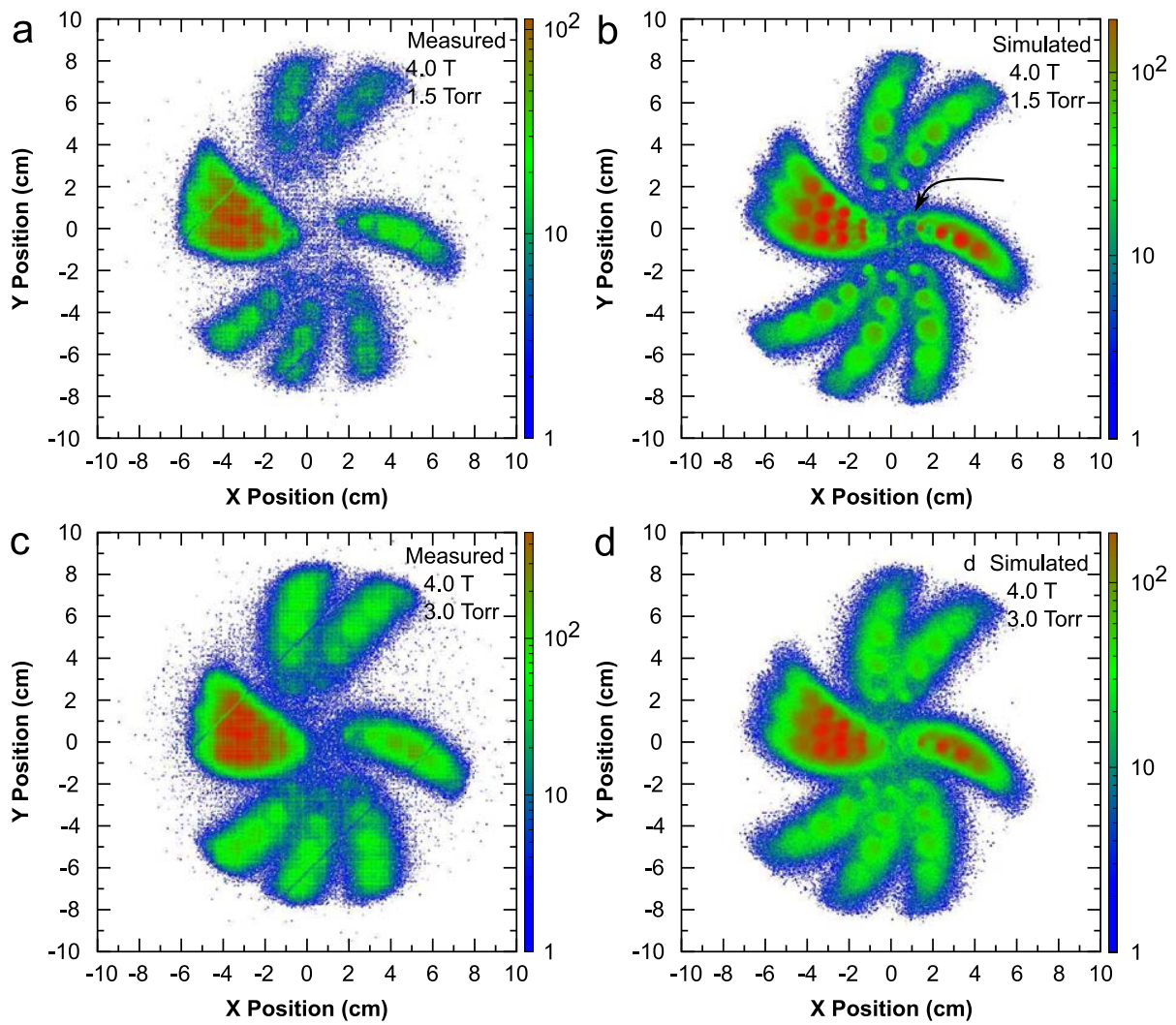

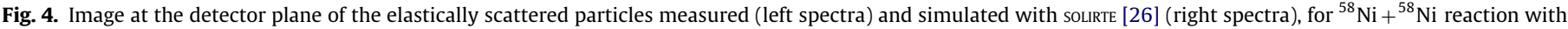
$E_{L a b}=220 \mathrm{MeV}$, at different $B_{z}$ and different He pressure in the gas cell.

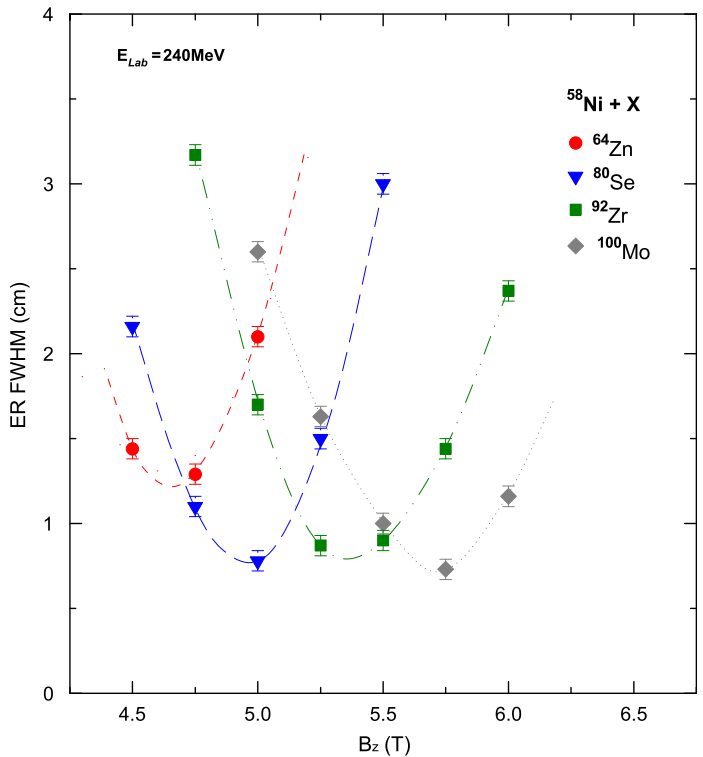

Fig. 5. Image size of the ERs on the MwPC for fusion of $240 \mathrm{MeV}^{58} \mathrm{Ni}$ for a range of targets, measured as a function of magnetic field. The lines are to guide the eye.

with the experimental data, as shown in Fig. 6 for ${ }^{58} \mathrm{Ni}+{ }^{74} \mathrm{Ge}$. The calculation with SOLIRTE (dashed line) using the optimal (empirical) $\langle q\rangle$ (as shown in Fig. 7) gives a good reproduction of the experimental image size versus magnetic field. Fig. 7 shows the empirical mean ER charge state obtained in this work as a function of the charge of the compound nucleus $\left(Z_{C N}\right)$.

Significant differences were found between the average charge state $\langle q\rangle$ predicted by simple models, and those obtained

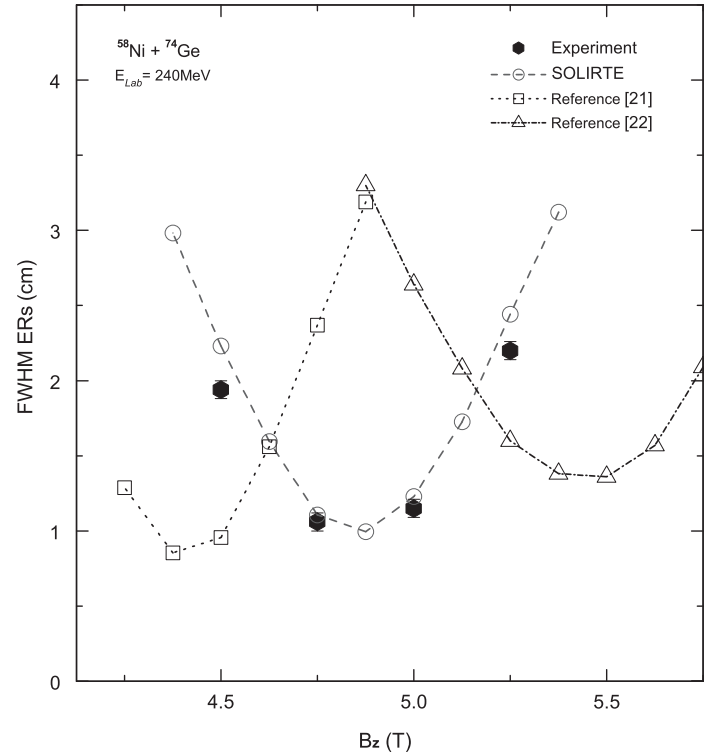

Fig. 6. Image size of the ERs on the MwPC for fusion of $240 \mathrm{MeV}{ }^{58} \mathrm{Ni}$ with a ${ }^{74} \mathrm{Ge}$ target. Calculated values are shown from different $\langle q\rangle$ predictions $[21,22]$ as a function of the magnetic field. The lines are to guide the eye.

empirically. As shown in Fig. 7, predictions using Eq. (5.7) from Ref. [21] systematically overestimate $\langle q\rangle$ for all the cases studied, whilst predictions from Ref. [22] underestimate $\langle q\rangle$, though with a decreasing difference for larger charges.

Because of the complex dependence of the charge state on the electronic structure of the ion, its velocity, and the gas it is passing through, measurement of the mean charge for each reaction appears to be the optimal approach at this stage to allow 
calculation of the trajectories. With the final detector arrangement, using two MwPCs, allowing ion track reconstruction, the axial distance of the focus (minimum image size) will be easily determined for each measurement. Hence the charge state, trajectories, and thus efficiency of the separator can be determined (using SOLIRTE) from the data collected during the fusion cross-section measurements.

\section{Identification of ERs}

A number of detectors and configurations were tested to identify evaporation residues, and separate them from the residual flux of beam particles reaching the detector position.

\subsection{Single MWPC detector}

Because of the availability of a pulsed beam from the ANU 14UD tandem electrostatic accelerator, with width $1 \mathrm{~ns}$ and separated by $106 \mathrm{~ns}$ or multiples thereof, ERs could be identified

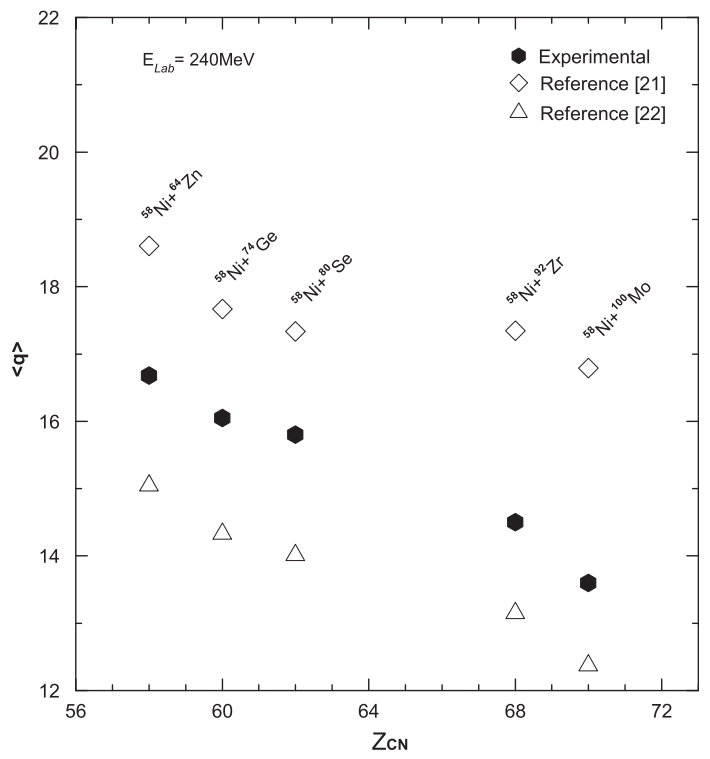

Fig. 7. Experimental and predicted mean charge state $\langle q\rangle$ versus $Z_{C N}$ for fusion products from each reaction. with a single detector, using time-of-flight and energy loss information.

For the reaction of ${ }^{58} \mathrm{Ni}$ with ${ }^{60} \mathrm{Ni}$, the typical ToF from the target to the detector $(168 \mathrm{~cm})$ is $60 \mathrm{~ns}$ for the elastically scattered beam, and $120 \mathrm{~ns}$ for ERs. Therefore the events are well separated in time. There are also energy-degraded beam particles which pass through the separator with the same flight time as the ERs. These can be rejected by the energy loss signal from the MWPC. Fig. 8(a) shows $\Delta E$ as a function of TOF for the reaction ${ }^{58} \mathrm{Ni}+{ }^{60} \mathrm{Ni}$ at $E_{L a b}=206 \mathrm{MeV}$. The field $B_{z}$ was $5.5 \mathrm{~T}$, and the separator filled with 1 Torr of He gas. The ERs are well-separated from beam particles, allowing accurate determination of the number of ER events.

If the ER charge state in gas is known, the $\mathrm{x}$ and $\mathrm{y}$ position information from the MWPC gives information on the angular distribution of the ERs leaving the target. The angular distribution is necessary to obtain an accurate calculation of the transmission efficiency of Solitaire. In the fusion measurements made up to now, angular distributions were available in the literature, which were sufficient to give calculated transmission efficiencies to better than $\sim 3 \%$. For even better accuracy, information on the angular distribution of the ERs emerging from the target can be determined from the position distribution measured in the MWPC, making use of the simulation program soliRTE. The method and validation of this procedure is beyond the scope of this paper, and will be described elsewhere.

\subsection{Two MWPC detectors}

Detailed investigation of sources of background showed that energy-degraded beam particles were produced as a result of interactions of the primary and/or scattered beam with solid surfaces both upstream and downstream from the target. The тоF measurement with a pulsed beam and a single detector cannot separate this class of events from interactions at the target location. Assuming all interactions occur at the target, the apparent velocities from the TOF spectra are thus incorrect for some events, which can lead to poorer separation of the ERs from the beam particle background.

Using two MWPC detectors, giving the TOF between fixed positions, allows true velocity determination (as well as eliminating the requirement of a pulsed beam), and permits trajectory reconstruction if necessary, to give an additional observable to reject beam particles. The detectors are normally $24 \mathrm{~cm}$ apart, which is quite sufficient to separate elastically scattered beam particles from the ERs. The first use of the two detectors together,
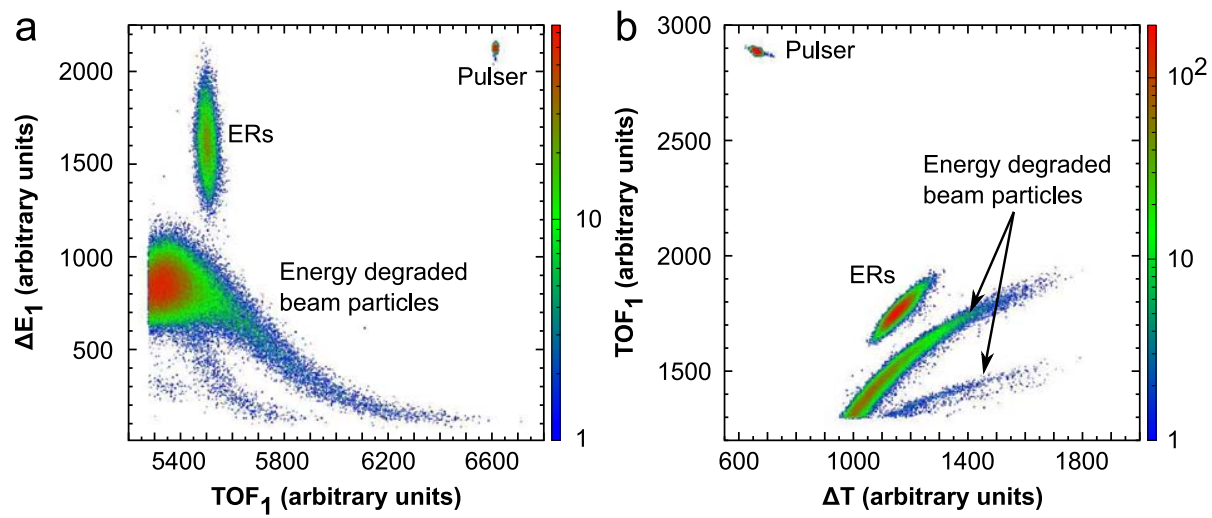

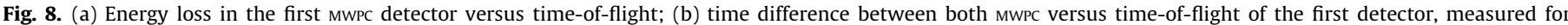
${ }^{58} \mathrm{Ni}+{ }^{60} \mathrm{Ni}$ for $E_{\text {Lab }}=220 \mathrm{MeV}$. 
in conjunction with a pulsed beam, revealed an additional benefit. The second TOF measurement between the two MwPC detectors ( $\Delta t=$ tof $_{2}$-tof ${ }_{1}$ ) was compared with the toF to the first detector tof ${ }_{1}$. Because of the energy loss of the particles, largely through the entry window and timing foil of the first MWPC, the velocities of all particles are reduced, but those of the ERs proportionately more than those of beam particles. The two time measurements thus can give a rather precise measure of the total energy loss through the first detector. This energy loss is very much larger than the energy loss in the low pressure detector gas between the MwPc electrodes, from which the $\Delta E$ signal from the MwPC detectors is obtained. Thus the separation of ERs from beam particles can be better achieved using tof ${ }_{1}$ vs. $\Delta t$ than using tof ${ }_{1}$ vs. the direct $\Delta E$ signal from the MWPC detector. This is seen by comparing Fig. 8(b) showing $\Delta t$ vs. TOF for the same measurement as shown in Fig. 8(a), which clearly demonstrates the good separation of ERs from beam particles.

\section{Fusion measurements}

\subsection{Validation of the ER transport efficiency calculation}

The availability of ER angular distribution measurements over a wide angular range, from close to $0^{\circ}$ to $14^{\circ}$, and precision fusion cross-section data for the system ${ }^{34} \mathrm{~S}+{ }^{89} \mathrm{Y}$ [28], measured with the ANU velocity filter, made it an ideal case to test the simulation code SOLIRTE. Thus, the ERs from this reaction were measured using SolitAiRe at a beam energy of $124 \mathrm{MeV}$, with 1 Torr of helium along the flight path, and at three different magnetic fields, $B_{z}=4.75$, 5.00 and $5.25 \mathrm{~T}$. As the minimum ER image size had not been reached at the lowest field, the value of $\langle q\rangle=9.3 \pm 0.1$ was determined for the ERs by comparing the position distributions of ERs measured for the three magnetic fields. The ER transmission efficiencies for these measurements were calculated with SOLIRTE employing the ER angular distribution from Ref. [28], obtaining $(90.3 \pm 0.8) \%,(89.7 \pm 0.9) \%$ and $(86.6 \pm 0.9) \%$ for $B_{z}=4.75,5.00$ and $5.25 \mathrm{~T}$, respectively. These transmission efficiencies values, within uncertainties, agree with those required to match the $\sigma_{E R}$ value determined in Ref. [28].

\subsection{Fusion excitation functions}

SolitAIRE was used to make measurements of the fusion excitation functions for the reactions ${ }^{58} \mathrm{Ni}+{ }^{58,60,64} \mathrm{Ni}$ at energies around the fusion barrier. A pulsed beam of ${ }^{58} \mathrm{Ni}$ ( $1 \mathrm{~ns}$ wide beam bursts separated by $640 \mathrm{~ns}$ ) was incident on $60 \mu \mathrm{g} / \mathrm{cm}^{2}$ self supporting targets of ${ }^{58,60,64} \mathrm{Ni}$. The beam energy from the accelerator was defined with an accuracy of better than $0.1 \mathrm{MeV}$ [29]. The energy loss in the carbon entry foil of the gas cell, and in the filling gas prior to reaching the target was accurately determined by comparing the monitor detector energies with and without entry foil and He gas. In the first run, the simplest detector configuration was used, having one MWPC as the ER detector. Four surface barrier monitor detectors for normalization of the beam current were mounted in the iron cone at the entry to Solitaire. The ER yields were measured for $E_{L a b}$ from 165 to $250 \mathrm{MeV}$. Elastically scattered beam particles were rejected in the analog electronics by their time of flight. The typical data collection time for each point was 15 minutes, at a conservative data acquisition rate of $400 \mathrm{~Hz}$, collecting up to $10^{5}$ ERs. In the second run, two MWPC detectors were used, as described in Section 4.2.

Preliminary cross-sections are shown in Fig. 9, using transmission efficiencies for Solitaire determined from velocity-

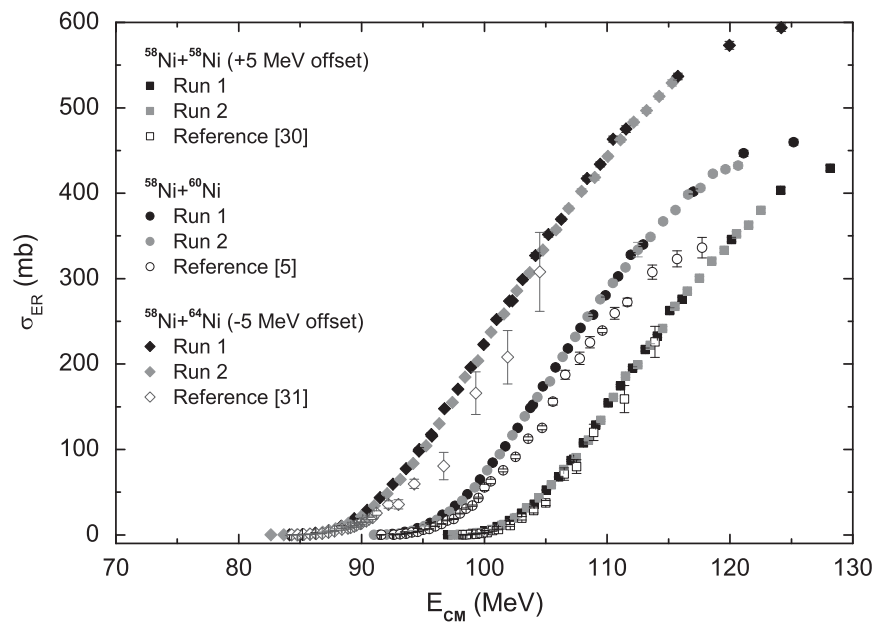

Fig. 9. Excitation functions for the reactions ${ }^{58} \mathrm{Ni}+{ }^{58,60,64} \mathrm{Ni}$ measured with Solitaire and with other devices, from Refs. [5,30,31]. Error bars where not shown are smaller than the size of the points. Offsets of $+5 \mathrm{MeV}$ and $-5 \mathrm{MeV}$ have been added to the ${ }^{58} \mathrm{Ni}+{ }^{58} \mathrm{Ni}$ and ${ }^{58} \mathrm{Ni}+{ }^{64} \mathrm{Ni}$ systems, respectively, for clarity.

scaled ER charge states based on the field-scanning measurements described in Section 3.3. Offsets of +5 and $-5 \mathrm{MeV}$ have been added to the ${ }^{58} \mathrm{Ni}+{ }^{58} \mathrm{Ni}$ and ${ }^{58} \mathrm{Ni}+{ }^{64} \mathrm{Ni}$ excitation functions, respectively, for clarity. The figure shows a number features of the new data:

(1) The scatter of the data is small, and appears to be consistent with the statistical uncertainties (typically $<1 \%$ ).

(2) There is a good agreement between the two different runs, with two different MWPC configurations.

(3) There is a change of slope at the highest energies for each reaction, this is attributed to increasing fission decay of the compound nuclei formed following fusion.

The figure also compares the data from this work with previously published results corresponding to the most extensive sets of data for these three reactions $[5,30,31]$. These previous measurements were made by scanning across the angular range of the ERs. The separating element was an electrostatic deflector in Ref. [5], which was followed by a time-of-flight telescope, whilst a velocity filter was used to separate the ERs in Refs. [30,31] which were subsequently detected using a $\Delta E-E$ telescope. Small systematic energy offsets between the present results and those of Refs. $[5,30,31]$ were found. Such energy offsets amongst different laboratories are not uncommon [32], as exemplified in Fig. 10 for the ${ }^{64} \mathrm{Ni}+{ }^{64} \mathrm{Ni}$ reaction, which has been previously measured at various laboratories elsewhere $[30,33,34]$. Agreement between the data sets for this reaction from different laboratories can only be achieved by energy offsets and/or changes in normalization.

From the excitation functions the experimental barrier distributions, $\mathrm{d}^{2}(E \sigma) / \mathrm{d} E^{2}$ were extracted using the point-difference procedure described in Ref. [4], with a $2 \mathrm{MeV}$ energy step [35]. Fig. 11 shows the barrier distribution for the ${ }^{58} \mathrm{Ni}+{ }^{60} \mathrm{Ni}$ reaction, together with that from the previous measurement [5], which used an electrostatic deflector followed by a time-of-flight telescope. The new barrier distribution shows less scatter of the data points than that of Ref. [5]. The scatter appears to be consistent with the error bars, which are calculated assuming the only uncertainties arise from the ER statistics obtained. Also shown is a coupled-channels calculation of the barrier distribution, assuming three quadrupole phonons in each nucleus, as described in Ref. [35]. This reproduces the general features of the measurement rather well. 


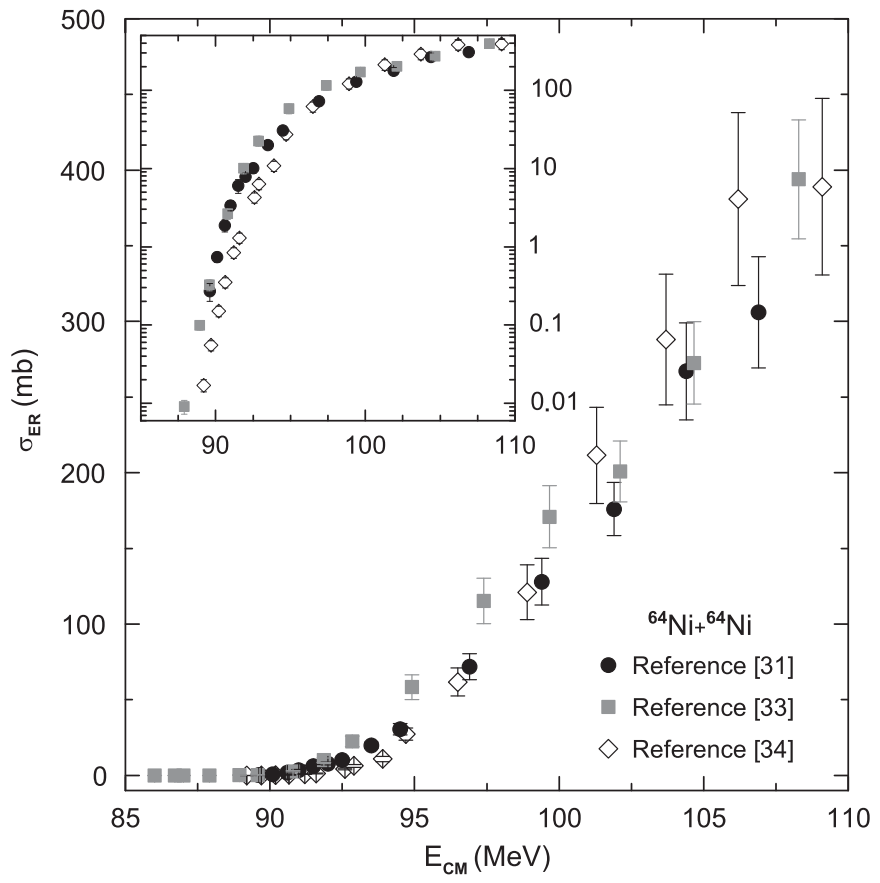

Fig. 10. Excitation functions for the reaction ${ }^{64} \mathrm{Ni}+{ }^{64} \mathrm{Ni}$ from Refs. $[31,33,34]$.

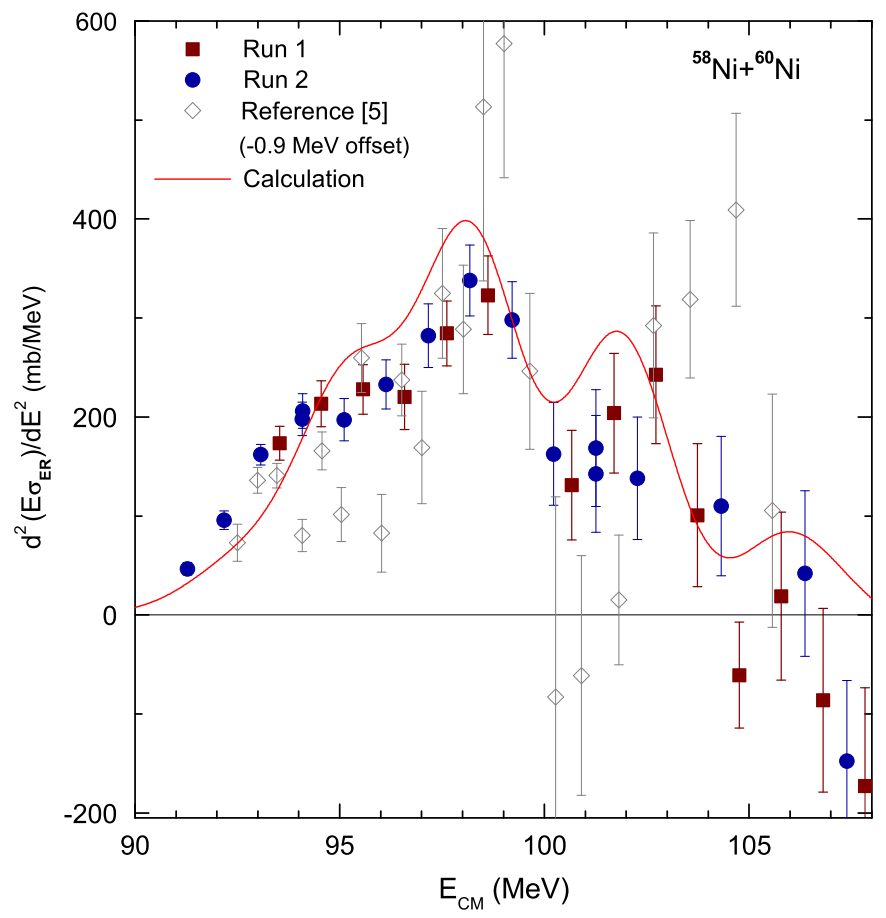

Fig. 11. The experimental barrier distribution for ${ }^{58} \mathrm{Ni}+{ }^{60} \mathrm{Ni}$ measured with SoltaIRE and from Ref. [5]. An offset of $-0.9 \mathrm{MeV}$ has been added to data from Ref. [5]. (See text for details).

\section{Boundaries to the use of SOLITAIRE}

There are three limitations in principle to the application of SolitAIRE to nuclear fusion reactions:

(1) Low energy ERs stop or scatter excessively in the gas for massasymmetric reactions with light projectiles;
(2) the charge states of ERs and beam particles can be too similar to give sufficient physical separation, for mass-symmetric reactions;

(3) the average charge state of ERs is so low that the maximum field of $6.5 \mathrm{~T}$ is insufficient to bring the ERs to a converging beam, which is likely to be a problem for low energy ERs formed in mass-asymmetric reactions.

All relate to the interactions of heavy ions with the filling gas, which are not yet well-understood. Thus the limitations in practice, namely for which reactions the gas-filled solenoidal separator will work successfully, and for which not, cannot yet be defined without further empirical investigations. If the physical basis for the actual behavior can be understood, and can be incorporated into the simulation code, then a realistic map of the operational limits of the device can be constructed.

The practical boundary due to limitation (1) can be addressed experimentally by investigating what is the lowest gas pressure that still brings low energy ERs to an acceptable focus. The boundary caused by limitation (2) has already been investigated in one case. As discussed in relation to the $\mathrm{Ni}+\mathrm{Ni}$ reactions, if the beam particles suffer almost no charge-changing collisions, the equilibrium charge state in gas is no longer relevant for the beam particles, giving a wider range of applicability to mass-symmetric reactions than was first expected. Limitation (3) is currently being investigated, through detailed studies of the most appropriate filling gas for a given reaction. Preliminary work indicates that the nature of the filling gas can change the ER charge state substantially, whilst having little effect on the beam particles. This work will be reported elsewhere.

\section{Conclusions and future directions}

A highly efficient ER separator SoltTAIRE, based on a gas filled $6.5 \mathrm{~T}$ superconducting solenoid, has been developed at the Australian National University. This compact device (flight path $\sim 1.8 \mathrm{~m}$ ) with a solid angle of $86 \mathrm{msr}$ provides a very high efficiency for ER measurements. A special feature is an iron return yoke which surrounds the solenoid to minimize the magnetic field at the target end, as well at the exit of the solenoid. The ERs exiting the solenoid are detected using large area position sensitive MwPCS detectors. The unmatched precision of the measurements of ERs following the reactions ${ }^{58} \mathrm{Ni}+{ }^{58,60,64} \mathrm{Ni}$ presented here, demonstrate the successful operation of this device.

The MWPC detectors cannot give unique mass identification of the ERs. Although this is not needed for fusion barrier distribution measurements, successful tests of implantation decay measurements, using a pulsed beam, looking at $\alpha$-particle decay of the ERs implanted into a Si detector, show that for $\alpha$-active nuclei, their identity can be readily determined by this technique. This technique also offers the possibility of making measurements with high selectivity for studies of quantum tunneling inhibition in heavy-ion fusion [36].

The wide angular acceptance and high transmission efficiency of the device is very attractive for coincidence measurements. For coincidences between ERs and particles evaporated from the hot compound nucleus, there is always an anti-correlation between the direction of the emitted particle, and that of the ER. This is one of the major physical effects determining the characteristics of the measured correlation when ERs are only detected with a small angular acceptance [37]. The very wide angular acceptance of Solitaire minimizes the effects of such correlations.

The device is also very suitable as a separator for nuclear structure studies, as the wide angular acceptance gives a high 
ER collection and transmission efficiency when thick (up to $1 \mathrm{mg} / \mathrm{cm}^{2}$ ) targets are used, even with relatively light projectiles, such as ${ }^{28} \mathrm{Si}[38]$. In conjunction with the short flight path, this confers unique advantages, which have led to the recent development of the $\gamma$-ray and conversion electron detector module SolEnoGam [38] which replaces the MWPC detector module behind the detector chamber. Physics measurements have already been carried out with this instrumentation [24].

The solenoid could also be used as a recoil mass separator [7,39] for $A<50$, where a combination of the magnetic focusing properties and measurement of energy and/or energy loss and time of flight information can give unique mass identification. Similar solenoids (singly or paired) have been applied to generate light radioactive ion beams by the in-flight transfer technique. The first was a Notre Dame University [40], whilst a very similar facility has been developed at the University of Sao Paulo [41]. Encouraged by these successes, developments are proceeding to produce a beam of ${ }^{6} \mathrm{He}$ at the ANU using SoltTaire. With the high voltage and beam intensity capability of the 14UD tandem accelerator at the ANU, there are a range of possible beams that will be investigated in future.

\section{References}

[1] C.H. Dasso, S. Landowne, A. Winther, Nucl. Phys. A 405 (1983) 381.

[2] N. Rowley, G.R. Satchler, P.H. Stelson, Phys. Lett. B 254 (1991) 25.

[3] J.R. Leigh, et al., Phys. Rev. C 47 (1993) R437.

[4] M. Dasgupta, et al., Annu. Rev. Nucl. Part. Sci. 48 (1998) 401.

[5] A.M. Stefanini, et al., Phys. Rev. Lett. 74 (1995) 864

[6] D.J. Hinde, et al., Phys. Rev. Lett. 74 (1995) 1295.
[7] H.A. Enge, Treatise on Heavy-Ion Science, vol. 7, Plenum Press, New York, London, 1984, p. 403.

[8] M. Leino, Nucl. Instr. and Meth. B 204 (2003) 129 and references therein.

[9] J.X. Wei, et al., Nucl. Instr. and Meth. A 306 (1991) 557.

[10] S. Beghini, et al., Nucl. Instr. and Meth. A 239 (1985) 585.

[11] J.P. Schapira, S. Gales, H. Laurent, IPN-Orsay Report, IPNO-PhN-7921, 1979.

[12] H. Laurent, J.P. Schapira, Nucl. Instr. and Meth. 162 (1979) 181.

[13] V.E. Cosslett, Introduction to Electron Optics, Clarendon Press, Oxford, 1950, pp. 77-147.

[14] H. Busch, Ann. Phys. 81 (1926) 974.

[15] H. Busch, Arch. Elektrotech. 28 (1927) 583.

[16] O. Klemperer, Electron Optics, second ed., Cambridge University Press, Cambridge, 1953, p. 443.

[17] A. Ghiorso, et al., Nucl. Instr. and Meth. A 269 (1988) 192.

[18] K.E. Rehm, et al., Nucl. Instr. and Meth. A 344 (1994) 614.

[19] M. Leino, et al., Nucl. Instr. and Meth. B 99 (1995) 653.

[20] P. Armbruster, et al., Nucl. Instr. and Meth. 91 (1971) 499.

[21] H.D. Betz, Rev. Mod. Phys. 44 (1972) 465.

[22] K.E. Gregorich, et al., Phys. Rev. C 72 (2005) 014605.

[23] I.S. Dimitriev, V.S. Nikolaev, Sov. Phys. JETP 20 (1964) 409.

[24] G.D. Dracoulis, et al., Phys. Rev. C 79 (2009) 031302R.

[25] K. Halbach, R.F. Holsinger, Part. Acceler. 7 (1976) 213.

[26] M.L. Brown, et al., to be published.

[27] M.L. Brown, Honours Thesis, The Australian National University, 2005, unpublished.

[28] A. Mukherjee, et al., Phys. Rev. C 66 (2002) 034607.

[29] C.R. Morton, et al., Phys. Rev. Lett. 72 (1994) 4074

[30] M. Beckerman, et al., Phys. Rev. C 23 (1981) 1581.

[31] M. Beckerman, et al., Phys. Rev. C 25 (1982) 837.

[32] C.R. Morton, et al., Phys. Rev. C 52 (1995) 243.

[33] C.L. Jiang, et al., Phys. Rev. Lett. 93 (2004) 012701.

[34] D. Ackermann, et al., Nucl. Phys. A 609 (1996) 91.

[35] M.D. Rodríguez, et al., AIP Conf. Proc. 853 (2006) 198.

[36] M. Dasgupta, et al., Phys. Rev. Lett. 99 (2007) 192701.

[37] D.J. Hinde, et al., Nucl. Phys. A 385 (1982) 109.

[38] P. Nieminen, et al., in: Nuclear Structure 2008 (NS2008), East Lansing, June 3-6, 2008.

[39] J.P. Schapira, et al., Nucl. Instr. and Meth. A 224 (1984) 337.

[40] M.Y. Lee, et al., Nucl. Instr. and Meth. A 422 (1999) 536.

[41] R. Lichtenthäler, et al., Nucl. Instr. and Meth. A 505 (2003) 612. 